\title{
Secondary Peripheral Chondrosarcoma
}

National Cancer Institute

\section{Source}

National Cancer Institute. Secondary Peripheral Chondrosarcoma. NCI Thesaurus. Code C121882.

A chondrosarcoma that arises close to the cartilaginous cap of a pre-existing osteochondroma. 